\title{
Migração, refúgio e saúde sexual e reprodutiva de mulheres na América Central, Sul e EUA: revisão integrativa
}

\author{
Migration, refuge and women's sexual and reproductive health in Central, South and USA: an \\ integrative review \\ Migración, refugio y salud sexual y reproductiva de las mujeres en Centro, Sur y Estados Unidos: \\ una revisión integradora
}

Joyce Veceli Barros da Sobreira

ORCID: https://orcid.org/0000-0002-2247-0586

Universidade Federal do Maranhão, Brasil E-mail: joyvcl181@gmail.com

Ester Godinho Sousa

ORCID: https://orcid.org/0000-0001-7972-5626

Universidade Federal do Maranhão, Brasil

E-mail: estergodinhosousa2@gmail.com

Lara Sobral Fiterman Lima

ORCID: https://orcid.org/0000-0001-7593-4084

Universidade Federal do Maranhão, Brasil E-mail: fitermanlara@gmail.com

Cesar Augusto Ferreira de Carvalho ORCID: https://orcid.org/0000-0001-8682-6417

Universidade Federal do Maranhão, Brasil

E-mail: cesar.augusto.f.carvalho@gmail.com

Pia Riggirozzi

ORCID: https://orcid.org/0000-0001-5809-890X

Universidade de Southampton, Reino Unido

E-mail: P.Riggirozzi@ soton.ac.uk

Natália Cintra de Oliveira Tavares

ORCID: https://orcid.org/0000-0003-3783-4300

Universidade de Southampton, Reino Unido E-mail: N.Cintra-De-Oliveira-Tavares@ soton.ac.uk

Zeni Carvalho Lamy

ORCID: https://orcid.org/0000-0002-9332-0542

Universidade Federal do Maranhão, Brasil

E-mail: zenilamy@gmail.com

Ruth Helena de Souza Britto Ferreira de Carvalho

ORCID: https://orcid.org/0000-0003-1180-1586

Universidade Federal do Maranhão, Brasil

E-mail: ruth.britto@gmail.com

Sara Fiterman Lima

ORCID: https://orcid.org/0000-0003-0015-3413

Universidade Federal do Maranhão, Brasil

E-mail: sara.fiterman@gmail.com

\begin{abstract}
Resumo
Trata-se de revisão cujo objetivo foi explorar evidências sobre as necessidades, os riscos e o acesso aos serviços de saúde sexual e reprodutiva entre mulheres migrantes ou refugiadas nos países da América Central, Sul e Estados Unidos (EUA). Estudo de revisão integrativa, cuja questão norteadora utilizada foi: "Qual é a situação relativa às necessidades, aos riscos e ao acesso aos serviços de saúde sexual e reprodutiva de mulheres latino-americanas migrantes deslocadas nos países da América Central, Sul e Estados Unidos?”. Foram realizadas buscas nas bases: PubMed, Cumulative Index for NursingandAllied Health Literature (CINAHL), Scientific Eletronic Library Online (SciELO) e Literatura Latino-Americana e do Caribe em Ciências da Saúde (LILACS), além de uma busca manual a partir das listas de referências dos artigos incluídos e no Google Scholar. Dos 910 estudos obtidos, 40 artigos compuseram a amostra final. Os achados foram descritos utilizando análise de conteúdo na modalidade temática. $\mathrm{O}$ processo de migração foi marcado por maior exposição e vulnerabilidade a traumas; houve predominância de barreiras para o acesso aos serviços de saúde pelas mulheres; destacou-se menor frequência de testagem de ISTs e chances aumentadas de comportamentos sexuais de risco; e percebeu-se disparidades nos cuidados obstétricos de
\end{abstract}


mulheres migrantes em comparação às mulheres dos países de acolhimento. A migração destas nos corredores da América Central e do Sul, apresenta considerável crescimento, no entanto ainda é pequeno o volume de produção para explicar o fenômeno nesses territórios.

Palavras-chave: Migração humana; Saúde sexual e reprodutiva; Direitos sexuais e reprodutivos.

\begin{abstract}
This review aimed to explore evidence on the needs, risks and access to sexual and reproductive health services among migrant or refugee women in Central, South and United States (USA). Integrative review study, whose guiding question was: "What is the situation regarding the needs, risks and access to sexual and reproductive health services of displaced Latin American migrant women in Central, South and United States countries?". Searches were performed in the following data bases: PubMed, Cumulative Index for Nursing and Allied Health Literature (CINAHL), Scientific Electronic Library Online (SciELO) and Latin American and Caribbean Literature in Health Sciences (LILACS), in addition to a manual search from the reference lists of included articles and Google Scholar searches. Of the 910 studies obtained, 40 articles made up the final sample. The findings were described using content analysis in thematic modality. The migration process was marked by greater exposure and vulnerability to trauma; there was a predominance of barriers to women's access to health services; highlighted lower frequency of testing for STIs and increased chances of risky sexual behavior; and disparities in obstetric care of migrant women compared to women from host countries were noted. The migration of women in the corridors of Central and South America has shown considerable growth, however the volume of production to explain the phenomenon in these territories is still small.

Keywords: Human migration; Sexual and reproductive health; Reproductive rights.
\end{abstract}

\title{
Resumen
}

Esta revisión tuvo como objetivo explorar la evidencia sobre las necesidades, los riesgos y el acceso a los servicios de salud sexual y reproductiva entre las mujeres migrantes o refugiadas en los países del Centro, Sur y Estados Unidos. Estudio de revisión integradora, cuya pregunta orientadora fue: “¿Cuáles la situación respecto a las necesidades, riesgos y acceso a los servicios de salud sexual y reproductiva de mujeres migrantes latinoamericanas desplazadas en países de Centro, Sur y Estados Unidos?”. Las búsquedas se realizaron en las siguientes bases de datos: PubMed, Cumulative Index for Nursingand Allied Health Literature (CINAHL), Scientific Electronic Library Online (SciELO) y LatinoAmericana Caribbean Literature in Health Sciences (LILACS), además de una búsqueda manual de las listas de referencias de los artículos incluidos y las búsquedas de Google Académico. De los 910 estudios obtenidos, 40 artículos conformaron la muestra final. Los hallazgos se describieron mediante análisis de contenido en modalidad temática. El proceso de migración estuvo marcado por una mayor exposición y vulnerabilidad al trauma; predominaron las barreras al acceso de las mujeres a los servicios de salud; destacó la menor frecuencia de las pruebas de detección de ITS y el aumento de las posibilidades de comportamientossexuales de riesgo; y se observaron disparidades en la atención obstétrica de las mujeres migrantes en comparación con las mujeres de los países de acogida. La migración de mujeres en los corredores de Centro y Sudamérica ha mostrado un crecimiento considerable, sin embargo el volumen de producción para explicar el fenómeno en estos territorios aún pequeño. Palabras clave: Migración humana; Salud sexual y reproductiva; Derechos sexuales y reproductivos.

\section{Introdução}

A saúde sexual e reprodutiva (SSR) das populações tornou-se um imperativo moral no âmbito dos direitos humanos. Para as pessoas em situação de migração e refúgio, este contexto surte efeito desproporcional na saúde dos mais pobres e vulneráveis, principalmente entre mulheres adultas e adolescentes (WHO, 2012), as quais correspondem a quase metade (48\%) dos 272 milhões de migrantes internacionais em todo o mundo (ONU, 2019).

Deflagrado um fenômeno migratório novo, os países da América Latina e do Caribe têm enfrentado uma das maiores crises humanitárias da região, haja vista os quase 5,4 milhões de venezuelanos fora de seu país de origem, sendo a maioria deslocada para os países vizinhos como Colômbia, Peru e Brasil (UNHCR, 2021). Em 2018, aproximadamente 340 mil venezuelanos pediram refúgio. Neste cenário, a migração intrarregional na América do Sul torna-se significativa pela intensificação recente do deslocamento dentro da sub-região. Da mesma forma, a migração para o Norte continua sendo tendência predominante na América Central, México e Caribe (McAuliffe et al., 2019).

O movimento migratório pode estar atrelado a eventos agudos, a instabilidade econômica, ou crises humanitárias, como também a eventos a longo prazo. Sobretudo, pode ser resultado de padrões de "corredores" de migrações desenvolvidos ao longo dos anos (McAuliffe et al., 2019). A migração feminina, por sua vez, pode ser motivada por diversos fatores além de 
questões financeiras, como falta de oportunidades na educação, para mulheres e seus filhos, no mercado de trabalho, e falta de acesso aos serviços públicos essenciais. A violência por parceiro íntimo (VPI) e violência generalizada também são reportadas como fatores que contribuem para essa migração (Dutra, 2013). Caso observado especificamente no Triângulo Norte da América Central (El Salvador, Guatemala, Honduras) e México, uma das regiões mais violentas do mundo controlada por grupos armados do crime organizado, muitas vezes com alcance transnacional, em que as altas taxas de assassinato e violência de gênero levaram milhares de mulheres e crianças a deixarem suas casas em 2017 (UNHCR, 2015).

Além disso, a migração pode levar a uma maior exposição a riscos, contudo, esta também pode estar associada à melhoria da saúde, principalmente para os migrantes que buscam segurança (McAuliffe et al., 2019). No entanto, condições sociais, doenças infecciosas, acesso restrito aos serviços de saúde, e barreiras culturais e linguísticas são fatores que contribuem para a deterioração da saúde dessas populações (Miramontes et al., 2015), adicionalmente, há ocorrência da limitação de conhecimentos sobre SSR, tanto entre adultas como adolescentes atrelados aos próprios desafios do processo migratório (Rivillas, 2018; Metusela et al., 2017).

A oferta de serviços e intervenções em SSR é imprescindível para a garantia da Saúde Sexual e Reprodutiva e dos Direitos (SSRD) na vida das mulheres migrantes. Evidencia-se a relevância desses serviços pelo impacto observado na mortalidade perinatal (Minsart et al., 2013), nos abortos espontâneos e natimortos (Sturrock et al., 2020), e na menor utilização de métodos contraceptivos, associada à ocorrência de gravidez não desejada e a sua interrupção voluntária, indicados pelo baixo uso de planejamento familiar (20,6\%) quando comparado à média regional do país de acolhimento de 70\% (Mendoza \& Miranda, 2019). Ademais, as imigrantes se encontram em situações de maior probabilidade para a adoção de comportamentos de risco com relação à transmissão de doenças infeciosas, como o HIV/AIDS. A migração feminina também revela perigos, como exploração sexual e condições de trabalho, estes se tornam agravantes à SSR (Gabaccia, 2016; Rocha-Jimenez et al., 2018).

Diante disso, as migrantes são particularmente vulneráveis a disparidades de saúde sexual e reprodutiva. Entretanto, com relação à interseção de gênero com status migratório e étnico, há poucos relatos na literatura acerca das implicações dessas experiências na SSR ou no bem-estar dessas migrantes. Além disso, destaca-se poucos estudos em profundidade sobre saúde, gênero e migração internacional (Ivanova et al., 2018; Alarcão, Stefanovska-Petkovska et al., 2021). Isto fica ainda mais evidente no caso das migrantes na América Latina. Portanto, esta revisão se propõe a explorar evidências sobre necessidades, riscos e acesso aos serviços de saúde sexual e reprodutiva entre mulheres migrantes nos países da América Central, Sul e Estados Unidos.

\section{Método}

Estudo de revisão integrativa, cujo método foi escolhido pelo potencial de capturar a complexidade de perspectivas amplas e variadas de um fenômeno estudado. $\mathrm{O}$ estudo seguiu as seguintes etapas: identificação do problema, pesquisa na literatura, avaliação de dados, análise de dados e apresentação (Whittemore \& Knafl, 2005). Adicionalmente, este estudo também se apoiou nas recomendações do Preferred Reporting Items for Systematic Reviews and Meta-Analysis PRISMA (Liberati et al., 2009).

Para a coleta dos dados, utilizou-se como questão norteadora: "Qual é a situação relativa às necessidades, aos riscos e ao acesso aos serviços de saúde sexual e reprodutiva de mulheres latino-americanas migrantes deslocadas nos países da América Central, Sul e Estados Unidos?”. Foram realizadas buscas nas bases: PubMed, Cumulative Index for Nursing and Allied Health Literature (CINAHL), Scientific Eletronic Library Online (SciELO) e Literatura Latino-Americana e do Caribe em Ciências da Saúde (LILACS). Além disso, realizou-se uma busca manual a partir das listas de referências dos artigos incluídos, assim feito também no Google Scholar. 
Adotados os termos e suas combinações (Quadro 1), as buscas foram limitadas para publicações de 2000 a 2020 , devido aos estudos atuais refletirem mais precisamente o contexto da migração contemporânea, e tomando como marco temporal a Declaração do Milênio das Nações Unidas, visto tratar-se de um documento histórico o qual discute questões de saúde e igualdade de gênero para um novo século. A última busca nas bases consultadas foi realizada em 29 de julho de 2020.

Quadro 1. Estratégia de busca na literatura a partir das bases escolhidas, São Luís - MA, 2020.

\begin{tabular}{|cl|c|}
\hline Base & \multicolumn{1}{c|}{ Termos de busca } \\
\hline PubMed & $\begin{array}{l}\text { ("Emigration and Immigration”[Mesh]) AND ("sexual health”[Mesh] OR "reproductive health"[Mesh] OR } \\
\text { contraception[ti] OR “family planning”[ti] OR pregnancy[ti] OR "prenatal care”[ti] childbirth[ti] OR "sexually } \\
\text { transmitted infection"[ti] OR HIV[ti] OR "maternal health”[Mesh]) }\end{array}$ \\
\hline CINAHL & $\begin{array}{l}\text { ("Emigration and immigration") AND ("sexual health" OR "reproductive health" OR "contraception" OR "family } \\
\text { planning" OR "pregnancy" OR "prenatal care" OR "childbirth" OR "sexually transmitted diseases" OR "Human } \\
\text { Immunodeficiency Virus" OR "Maternal-Child Health") }\end{array}$ \\
\hline LILACS & $\begin{array}{l}\text { (MH:"Emigração e Imigração") AND (MH:"saúde sexual" OR MH:"saúde reprodutiva" OR anticoncepção OR } \\
\text { planejamento familiar OR gravidez OR cuidado pré-natal OR parto OR Doenças sexualmente transmissíveis OR HIV } \\
\text { OR MH:"saúde materna") }\end{array}$ \\
\hline SciELO & $\begin{array}{l}\text { ("Emigração e imigração") AND ("saúde sexual" OR "saúde reprodutiva" OR (ti:(anticoncepção)) OR (ti:(planejamento } \\
\text { familiar)) OR (ti:(gravidez)) OR (ti:(cuidado pré-natal)) OR (ti:(parto)) OR (ti:(doenças sexualmente transmissíveis)) OR } \\
\text { (ti:(hiv)) OR ( "saúde materna") }\end{array}$ \\
\hline
\end{tabular}

Fonte: Autores (2020).

A população de interesse deste estudo é de mulheres e adolescentes migrantes ou refugiadas. Adotamos as definições oficiais da Organização das Nações Unidas (ONU) e da Organização Internacional para Imigração (IOM) para migrantes e refugiados (IOM, 2012). Não abordaremos imigrantes de segunda geração devido ao estudo voltar-se diretamente à observação dos impactos do fluxo migratório na SSR no país de acolhimento.

As buscas foram feitas sem limitação de idioma que abordavam a temática da saúde sexual e reprodutiva: (a) cuidados pré-natais, durante o parto, puerpério e cuidados obstétricos de emergência; (b) aconselhamento e serviços de contracepção; (c) serviços de aborto seguro e tratamento de complicações relacionadas; e (d) prevenção e tratamento do vírus da imunodeficiência humana (HIV) e outras infecções sexualmente transmissíveis (IST) (Starrs et al., 2018).

Foram excluídos (a) duplicatas; (b) estudos considerados literatura cinzenta (editoriais, teses e anais); (c) publicações cujos participantes não eram mulheres adultas ou adolescentes latino-americanas migrantes ou em situação de refúgio nos países da América Central, Sul e Estados Unidos; (d) estudos que tratavam de migração interna; (e) revisões de literatura; (f) imigrantes de segunda geração; e (g) desfechos no recém-nascido.

Dos 905 artigos obtidos na busca nas bases, acrescido de cinco artigos da busca manual, 85 duplicatas foram identificadas. As duplicações foram removidas usando a estratégia de identificação de duplicatas do software Mendeley (Gunn, 2014), após os artigos serem exportados das bases de dados pesquisadas.

Para triagem dos artigos, foi utilizado o Rayyan (Ouzzani et al., 2016) e aplicado o processo de revisão por pares. A exclusão dos estudos ocorreu a partir da leitura dos títulos e dos resumos dos 825 selecionados, dentre eles, 762 artigos foram excluídos e 63 foram selecionados para a leitura na íntegra. Os artigos que respondiam à questão norteadora foram incluídos na amostra final correspondendo a 40 no total. O processo de seleção dos artigos incluídos nessa revisão é apresentado em fluxograma PRISMA (Figura 1). 
Figura 1. Fluxograma de etapas empregadas para seleção da amostra, São Luís - MA, 2020.

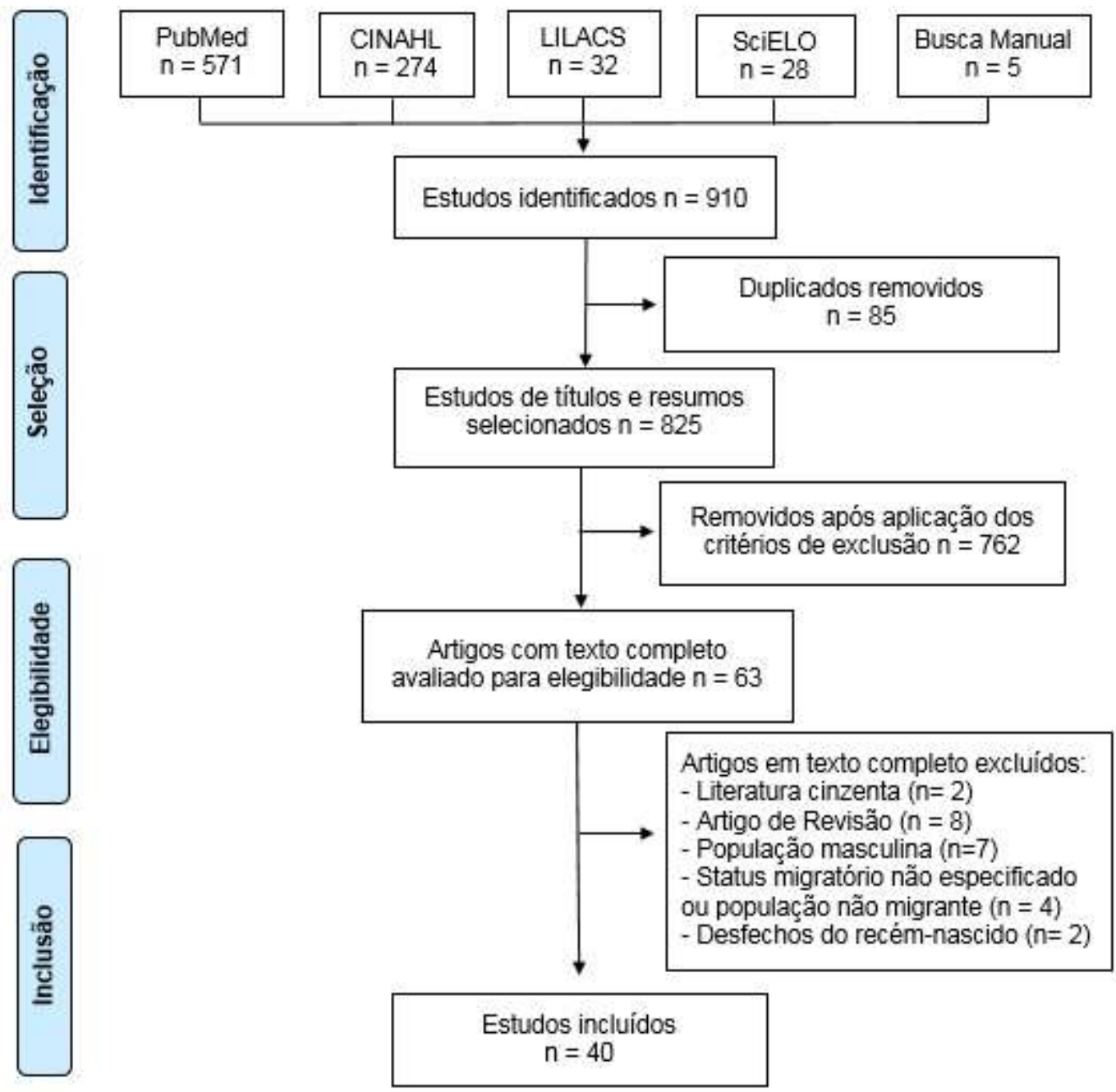

Fonte: Autoria (2020).

As três primeiras autoras leram independentemente os artigos incluídos na triagem e realizaram a extração dos dados para um formulário específico criado para reunir: autor, ano de publicação, país de publicação, objetivos, desenho do estudo, origem da imigrante, e Nível de Evidência (NE) descrito por Stetler et al. (1988): nível I - meta-análises de estudos clínicos controlados e randomizados; nível II - estudo de desenho experimental; nível III - estudos quase-experimentais; nível IV estudos não experimentais qualitativos ou estudos descritivos; nível V - relato de casos ou relatos de experiências; e nível VI opiniões de especialistas ou com base em normas regulamentadoras ou legais.

Para organização das informações foram diferenciados os estudos de abordagem quantitativa, qualitativa e de métodos mistos. Em continuidade, realizou-se uma síntese narrativa dos dados utilizando análise temática (Bardin, 2011).

\section{Resultados}

Esta revisão encontrou 40 publicações (Quadro 2) descritas segundo sua autoria, ano de publicação, país, objetivo principal, delineamento, origem e caracterização do imigrante apresentados pelos artigos. 
Considerando o autor de correspondência como autor principal, foi constatada predominância do gênero feminino (32), com maioria tendo origem nos EUA (29), seguido por Brasil (6), Chile (2), e Colômbia, Costa Rica e Guatemala com um artigo cada.

Com relação ao tipo de estudo, foram identificados com abordagem quantitativa (27), sendo eles, delineamento transversal (21), coorte retrospectiva (2), descritivo (1), prospectivo (1), retrospectivo documental (1), e pré-pós-teste com grupo controle (1). Os estudos identificados com abordagem qualitativa (12), foram descritivos e analíticos (7), teorias fundamentadas e etnografias (4), e descritivo e exploratório (1). Apenas um estudo de método misto foi identificado (a abordagem qualitativa tinha como delineamento a história de vida, e a quantitativa, longitudinal). A maioria dos artigos era não experimental (39), nível de evidência IV, sendo um quase-experimental, nível de evidência III.

Com relação aos países de origem das imigrantes, alguns estudos especificaram esses países (36) e outros generalizaram essa população como "latina" ou "hispânica" (4). A idade das participantes foi apresentada em 38 artigos, entre eles alguns estudos abordaram apenas mulheres adultas na faixa de 18 a 64 anos (16), adolescentes com faixa etária de 13 a 19 anos (2), estudaram tanto adultas quanto adolescentes na faixa etária de 13 a 64 anos (20), e artigos não informaram a idade dos participantes (2).

Alguns estudos combinaram os resultados de todas as mulheres nascidas no exterior com as nascidas no país de acolhimento em sua análise (5), ou os estratificaram apenas por raça: latina (2). As publicações se referiam às mulheres imigrantes, sendo a maioria proveniente do México (23), seguido por Caribe (10), EUA (6), El Salvador (6), Bolívia (5), Colômbia (5), Honduras (5), Guatemala (5), Equador (3), Nicarágua (3), Peru (3), Venezuela (2), Austrália, Argentina, Brasil, Canadá, Costa Rica, Espanha, Fiji, Filipinas, Paraguai, Uruguai e Vietnã com um artigo em cada. Dentre os artigos, houve (5) os que privilegiaram a descrição de uma divisão mais abrangente (África, América Central, América do Sul, Ásia, Europa, Subcontinente Indiano e Sudeste da Ásia), e outros (4) que não informaram o país de origem. Mais detalhes estão descritos no Quadro 2. 
Research, Society and Development, v. 10, n. 16, e5101623698, 2021

(CC BY 4.0) | ISSN 2525-3409 | DOI: http://dx.doi.org/10.33448/rsd-v10i16.23698

Quadro 2. Caracterização dos estudos incluídos, São Luís - MA, 2021.

\begin{tabular}{|c|c|c|c|c|c|c|}
\hline No. & Autor, Ano & País & Objetivo & Desenho/ NE & Caracterização da imigrante & Origem da imigrante \\
\hline \multicolumn{7}{|c|}{ Estudos quantitativos } \\
\hline 1 & Althoff et al. (2017) & EUA & $\begin{array}{l}\text { Descrever a quantidade e a qualidade das redes de apoio social de } \\
\text { imigrantes latinos e o papel que essas redes desempenham em seus } \\
\text { comportamentos de risco para HIV / DST }\end{array}$ & Transversal/ IV & $\begin{array}{l}\text { Idade: ( } 36,3 \text { anos }) \\
43 \% \text { mulheres }\end{array}$ & $\begin{array}{l}\text { Honduras, México, } \\
\text { Guatemala, Nicarágua, } \\
\text { Costa Rica, República } \\
\text { Dominicana, Cuba } \\
\end{array}$ \\
\hline 2 & Bahamondes et al. (2020) & Brasil & $\begin{array}{l}\text { Fornecer uma visão geral das questões de SSR que afetam mulheres } \\
\text { migrantes venezuelanas no estado de Roraima, Brasil }\end{array}$ & Transversal/ IV & $\begin{array}{l}\text { Idade: } 18-49 \text { anos } \\
(30,1 \text { anos) } \\
\% \text { mulheres* }\end{array}$ & Venezuela \\
\hline 3 & $\begin{array}{l}\text { Batista, Gugelmin \& Muraro } \\
(2018)\end{array}$ & Brasil & $\begin{array}{l}\text { Analisar o acompanhamento pré-natal realizado pelas gestantes haitianas } \\
\text { e brasileiras residentes no estado de Mato Grosso }\end{array}$ & Transversal/ IV & $\begin{array}{l}\text { Idade: }<25 \text { anos; } 25-29 \text { anos; } 30 \\
\text { anos }< \\
\% \text { mulheres* }\end{array}$ & Haiti \\
\hline 4 & $\begin{array}{l}\text { Bautista et al. } \\
(2007)\end{array}$ & EUA & $\begin{array}{l}\text { Comparar comportamentos de risco relacionados ao HIV de imigrantes } \\
\text { com não-imigrantes entre mulheres trabalhadoras do sexo na América } \\
\text { do Sul }\end{array}$ & Transversal/ IV & $\begin{array}{l}\text { Idade: } 18-24 \text { anos } \\
\% \text { mulheres* }\end{array}$ & $\begin{array}{l}\text { República Dominicana, } \\
\text { Paraguai, Uruguai, } \\
\text { Equador, Brasil, Colômbia }\end{array}$ \\
\hline 5 & $\begin{array}{l}\text { Ceballos \& Palloni } \\
(2010)\end{array}$ & EUA & $\begin{array}{l}\text { Avaliar a hipótese do paradoxo de aculturação que atribui a deterioração } \\
\text { da saúde aos efeitos negativos da aculturação, enquanto viviam nos } \\
\text { EUA }\end{array}$ & Transversal/ IV & $\begin{array}{l}\text { Idade: }(26,6 \text { anos }) \\
\% \text { mulheres* }\end{array}$ & México \\
\hline 6 & Fernández-Niño et al. (2019) & Colômbia & $\begin{array}{l}\text { Caracterizar a situação de saúde das gestantes migrantes venezuelanas } \\
\text { irregulares, residentes no Caribe Colombiano }\end{array}$ & Transversal/ IV & $\begin{array}{l}\text { Idade: } 14 \text { - } 41 \text { anos ( } 23,9 \text { anos) } \\
\% \text { mulheres* }\end{array}$ & Venezuela \\
\hline 7 & $\begin{array}{l}\text { Forna, Jamieson, Sanders \& Lindsay } \\
\text { (2003) }\end{array}$ & EUA & $\begin{array}{l}\text { Comparar os resultados da gravidez entre mulheres nascidas no exterior } \\
\text { e mulheres nascidas nos Estados Unidos }\end{array}$ & $\begin{array}{l}\text { Coorte } \\
\text { retrospectiva/ IV }\end{array}$ & $\begin{array}{l}\text { Idade: }(24,9 \text { anos }) \\
\% \text { mulheres* }\end{array}$ & $\begin{array}{l}\text { África, Asia, Caribe, } \\
\text { América Central e Sul, } \\
\text { México, El Salvador, } \\
\text { Europa, Canadá, Fiji, } \\
\text { Austrália }\end{array}$ \\
\hline 8 & Fortuna et. al. (2019) & EUA & $\begin{array}{l}\text { Examinar os fatores que têm impacto na saúde reprodutiva e emocional } \\
\text { das latinas imigrantes }\end{array}$ & Transversal/ IV & $\begin{array}{l}\text { Idade: } 18-64 \text { anos }(25,4 \text { anos }) \\
\% \text { mulheres* }\end{array}$ & $\begin{array}{l}\text { El Salvador, Guatemala, Porto } \\
\text { Rico, EUA, República } \\
\text { Dominicana, Colômbia, } \\
\text { México e América do Sul }\end{array}$ \\
\hline 9 & Fuentes-Afflick et al. (2006) & EUA & $\begin{array}{l}\text { Comparar o efeito do estado de residência e o status de imigração nos } \\
\text { cuidados pré-natais entre hispânicas no período após a promulgação do } \\
\text { PRWORA }\end{array}$ & Transversal/ IV & $\begin{array}{l}\text { Idade: } \geq 17 \text { anos } \\
\% \text { mulheres* }\end{array}$ & $\begin{array}{l}\text { México, Cuba, República } \\
\text { Dominicana, América } \\
\text { Central, América do Sul, } \\
\text { outros }\end{array}$ \\
\hline 10 & $\begin{array}{l}\text { Fullerton, Nelson, Shannon \& Bader } \\
\text { (2004) }\end{array}$ & EUA & $\begin{array}{l}\text { Investigar o impacto de um sistema de atendimento gerenciado pelo } \\
\text { Medicaid no acesso ao atendimento pré-natal }\end{array}$ & Transversal/ IV & $\begin{array}{l}\text { Idade: } 14-44 \text { anos (média } 25,4 \\
\text { anos) } \\
\% \text { mulheres* }\end{array}$ & NI \\
\hline
\end{tabular}


Research, Society and Development, v. 10, n. 16, e5101623698, 2021

(CC BY 4.0) | ISSN 2525-3409 | DOI: http://dx.doi.org/10.33448/rsd-v10i16.23698

\begin{tabular}{|c|c|c|c|c|c|c|}
\hline 11 & Jones, Kubelka \& Bond (2001) & EUA & $\begin{array}{l}\text { Descrever o estado de aculturação, os índices demográficos e de } \\
\text { gravidez selecionados e a relação com os resultados do nascimento e } \\
\text { padrões de planejamento familiar }\end{array}$ & Transversal/ IV & $\begin{array}{l}\text { Idade: } 13-19 \text { anos } \\
(18 \text { anos }) \\
\% \text { mulheres* }\end{array}$ & México \\
\hline 12 & $\begin{array}{l}\text { Jones, Bond, Gardner \& Hernandez } \\
\text { (2002) }\end{array}$ & EUA & $\begin{array}{l}\text { Descrever o planejamento familiar de mulheres hispânicas e determinar } \\
\text { sua relação com o nível de aculturação e com indicadores demográficos } \\
\text { maternos selecionados }\end{array}$ & Transversal/ IV & $\begin{array}{l}\text { Idade: }(24,3 \text { anos }) \\
\% \text { mulheres* }\end{array}$ & México \\
\hline 13 & Joyce, Bauer, Minkoff \& Kaestner (200 & EUA & $\begin{array}{l}\text { Analisar as mudanças no financiamento do cuidado pré-natal e do parto, } \\
\text { entre mulheres latinas nascidas no exterior e nos EUA após a } \\
\text { promulgação da Lei de Reconciliação de Responsabilidade Pessoal e } \\
\text { Oportunidades de Trabalho }\end{array}$ & $\begin{array}{l}\text { Pré-pós teste com grupo } \\
\text { controle/ } \\
\text { III }\end{array}$ & $\begin{array}{l}\text { Idade: NI } \\
\% \text { mulheres* }\end{array}$ & $\begin{array}{l}\text { México, República } \\
\text { Dominicana, Outras latinas }\end{array}$ \\
\hline 14 & $\begin{array}{l}\text { Kilmer, Barker, Ly } \\
\& \text { Jiles (2019) }\end{array}$ & EUA & $\begin{array}{l}\text { Determinar se a vacinação contra hepatite B e a prevalência de triagem } \\
\text { diferem entre mulheres nascidas nos EUA e estrangeiras em idade } \\
\text { reprodutiva }\end{array}$ & Transversal/ IV & $\begin{array}{l}\text { Idade: } 18-44 \text { anos }(33,0 \text { anos }) \\
\% \text { mulheres* }\end{array}$ & $\begin{array}{l}\text { México, América Central, } \\
\text { illas do Caribe; Sudeste da } \\
\text { Ásia; Ásia, Europa; África; } \\
\text { América do Sul }\end{array}$ \\
\hline 15 & $\begin{array}{l}\text { Leal-Mateos \& } \\
\text { Barboza-Retana (2008) }\end{array}$ & Costa Rica & $\begin{array}{l}\text { Examinar as características do controle pré-natal e do parto na } \\
\text { população imigrante que dá à luz no Hospital HCG, Costa Rica }\end{array}$ & Transversal/ IV & $\begin{array}{l}\text { Idade: }(27,3 \text { anos }) \\
\% \text { mulheres* }\end{array}$ & $\begin{array}{l}\text { Nicarágua, Colômbia, El } \\
\text { Salvador, Honduras, Peru, } \\
\text { Equador, Espanha }\end{array}$ \\
\hline 16 & $\begin{array}{l}\text { Loue, Cooper \& } \\
\text { Fiedler (2003) }\end{array}$ & EUA & $\begin{array}{l}\text { Analisar o risco de contaminação por HIV em uma amostra de } \\
\text { imigrantes mexicanos e porto riquenhos nos EUA }\end{array}$ & Transversal/ IV & $\begin{array}{l}\text { Idade: }(30,9 \text { anos }) \\
45 \% \text { mulheres }\end{array}$ & México e Porto Rico \\
\hline 17 & $\begin{array}{l}\text { Loue, Cooper \& } \\
\text { Lloyd (2005) }\end{array}$ & EUA & $\begin{array}{l}\text { Avaliar as relações entre adesão ao pré-natal por mulheres mexicanas e } \\
\text { diversos status de migração no Condado de San Diego, Califórnia }\end{array}$ & Transversal/ IV & $\begin{array}{l}\text { Idade: }(32,8 \text { anos }) \\
\% \text { mulheres* }\end{array}$ & México \\
\hline 18 & Matsuda et al. (2014) & EUA & $\begin{array}{l}\text { Examinar as associações entre os fatores de relacionamento / } \\
\text { planejamento familiar e o poder do relacionamento sexual (SRP) }\end{array}$ & Transversal/ IV & $\begin{array}{l}\text { Idade: }(26,5 \text { anos }) \\
50 \% \text { mulheres }\end{array}$ & $\begin{array}{l}\text { México, El Salvador, } \\
\text { Honduras, Guatemala }\end{array}$ \\
\hline 19 & $\begin{array}{l}\text { McDonald, Suellentrop, } \\
\text { Paulozzi \& Morrow (2008) }\end{array}$ & EUA & $\begin{array}{l}\text { Comparar as características de saúde reprodutiva entre mães hispânicas e } \\
\text { não hispânicas brancas (NHW) }\end{array}$ & Transversal/ IV & $\begin{array}{l}\text { Idade: }<15,<20 \text { anos } \\
\% \text { mulheres* }\end{array}$ & NI \\
\hline 20 & Nuttbrock \& Hwahng (2017) & EUA & $\begin{array}{l}\text { Examinar diferenças étnicas no trabalho sexual, comportamento sexual } \\
\text { de alto risco e incidentes de HIV / DST entre mulheres transexuais na } \\
\text { cidade de Nova York }\end{array}$ & Prospectivo/ IV & $\begin{array}{l}\text { Idade: } 19-59 \text { anos }(34,0 \text { anos }) \\
\% \text { mulheres transexuais*** }\end{array}$ & NI \\
\hline 21 & $\begin{array}{l}\text { Potter, White, Hopkins, } \\
\text { Amastae \& Grossman (2010) }\end{array}$ & EUA & $\begin{array}{l}\text { Comparar as motivações e experiências de usuárias de anticoncepcionais } \\
\text { orais que os obtiveram em farmácias mexicanas e nas clínicas de } \\
\text { planejamento familiar no Texas }\end{array}$ & Transversal/ IV & $\begin{array}{l}\text { Idade: } 18 \text { - } 44 \text { anos } \\
\% \text { mulheres* }\end{array}$ & México \\
\hline 22 & $\begin{array}{l}\text { Santos, Drezett \& } \\
\text { Alves (2015) }\end{array}$ & Brasil & $\begin{array}{l}\text { Identificar características sociodemográficas de migrantes bolivianas } \\
\text { com gestação decorrente de estupro, atendidas em serviço público de } \\
\text { referência para abortamento legal }\end{array}$ & $\begin{array}{l}\text { Retrospectivo e } \\
\text { documental/ IV }\end{array}$ & $\begin{array}{l}\text { Idade: } 13 \text { - } 34 \text { anos ( } 24 \text { anos) } \\
\% \text { mulheres* }\end{array}$ & Bolívia \\
\hline
\end{tabular}


Research, Society and Development, v. 10, n. 16, e5101623698, 2021

(CC BY 4.0) | ISSN 2525-3409 | DOI: http://dx.doi.org/10.33448/rsd-v10i16.23698

\begin{tabular}{|c|c|c|c|c|c|}
\hline 23 & $\begin{array}{l}\text { Spetz, Baker, Phibbs, Pedersen \& Tafoya } \text { EUA } \\
(2000)\end{array}$ & $\begin{array}{l}\text { Examinar se a aprovação da Proposta } 187 \text { da Califórnia teve um efeito } \\
\text { negativo sobre o uso de cuidados pré-natais e resultados de parto }\end{array}$ & $\begin{array}{l}\text { Coorte } \\
\text { retrospectiva/ IV }\end{array}$ & $\begin{array}{l}\text { Idade: }(25,0 \text { anos }) \\
\% \text { mulheres* }\end{array}$ & México, Filipinas, Vietnã \\
\hline 24 & Waldman (2011) & $\begin{array}{l}\text { Identificar a potencial existência de entraves ao } \\
\text { acesso aos serviços de saúde por parte de imigrantes bolivianas na } \\
\text { cidade de São Paulo }\end{array}$ & $\begin{array}{l}\text { Descritivo/ } \\
\text { IV }\end{array}$ & $\begin{array}{l}\text { Idade: } 20 \text { - } 37 \text { anos } \\
\% \text { mulheres* }\end{array}$ & Bolívia \\
\hline 25 & Wilson (2008) & $\begin{array}{l}\text { Caracterizar o processo de aculturação, avaliando como a gravidez e os } \\
\text { sentimentos sobre a gravidez mudaram ao longo das gerações de } \\
\text { migração }\end{array}$ & Transversal/ IV & $\begin{array}{l}\text { Idade: NI } \\
\% \text { mulheres* }\end{array}$ & México \\
\hline 26 & Wilson (2009) & $\begin{array}{l}\text { Explorar as diferenças no uso de anticoncepcionais entre mulheres de } \\
\text { origem mexicana ao longo das gerações de migração }\end{array}$ & Transversal/ IV & $\begin{array}{l}\text { Idade: } 15-44 \text { anos } \\
\% \text { mulheres* }\end{array}$ & México \\
\hline
\end{tabular}
27 White, Blackburn, Manzella, Welty \& EUA
Explorar as mudanças no uso de serviços de saúde com financiamento Ex́blico as ing financiamento público, por ingrantes latios, no Alabana, após a implementação da do Alabama. Transversal/ IV Idade: menor ou maior que 18 anos $\quad$ NI

\section{Estudos qualitativos}

28 Arcos, Vollrath, Sánchez \& Bailey (2018) (2013)

$30 \quad$ Grace et al. (2020)

Silveira (2009)
29 Biggs, Combellick, Arons \& Brindis

$31 \quad$ Madi, Cassanti \&

Chile

EUA

EUA

Brasil

Brasil

EUA

EUA para explorar como a imigração pode ter influenciado suas decisões de
Compreender a experiência de mulheres imigrantes no processo de maternidade e criação de bebês menores de 6 meses, no contexto de vulnerabilidade social no Chile

Descrever os contextos sociais de jovens imigrantes latinas grávidas, reprodução

Examenta coerca reproduiva e o uso de estretegias de seguranca e

Examinar a coerção reprodutiva e o uso de estratégias de segurança e redução de danos em uma amostra de mulheres latinas

Descritivo e

analítico/ IV

Descritivo e analítico/ IV

Descritivo e

Descritivo e
analítico/ IV

Compreender as concepções das imigrantes bolivianas, na região centra de São Paulo, sobre gestação nos contextos de família, de adaptação como imigrantes

Entender o acesso aos serviços públicos de saúde de uma população específica: os imigrantes bolivianos residentes na cidade de São Paulo, a partir de sua própria perspectiva

Explorar como as aspirações e expectativas educacionais e de emprego individuais e familiares afetaram a gravidez de adolescentes entre
mulheres latinas jovens

Examinar os contextos e as relações que ligam a violência por parceiro íntimo e os comportamentos sexuais de risco entre um grupo de latinas HIV positivo

IV
Idade: 18 - 40 anos

$\%$ mulheres*

Peru, Colômbia e outros

Idade: $15-19$ ano

$\%$ mulheres*

Idade: 20 - 40 anos (30,7 anos) $\%$ mulheres*

México, EUA

El Salvador, Guatemala,

Honduras

Descritivo e Idade: 18 - 29 anos Bolívia

analítico/ IV \% mulheres*

Bolívia

Descritivo e Idade: 20 - 30 ano

analítico/ IV $\quad 74 \%$ mulheres

Bolívia

Descritivo e exploratório/ Idade: 15 - 35 anos

$\%$ mulheres*

Latina; México, EUA

$34 \quad$ Moreno (2007)

HIV positivo

Porto Rico, América do

Sul, República Dominicana América Central ou México 
Research, Society and Development, v. 10, n. 16, e5101623698, 2021

(CC BY 4.0) | ISSN 2525-3409 | DOI: http://dx.doi.org/10.33448/rsd-v10i16.23698

\begin{tabular}{|c|c|c|c|c|c|c|}
\hline 35 & $\begin{array}{l}\text { Rocha-Jiménez, Brouwer, Silverman, } \\
\text { Morales-Miranda \& Goldenberg (2017) }\end{array}$ & Guatemala & $\begin{array}{l}\text { Explorar como a implementação de práticas de saúde pública em torno } \\
\text { do trabalho sexual moldam a prevenção e o atendimento ao HIV entre } \\
\text { mulheres trabalhadoras do sexo }\end{array}$ & $\begin{array}{l}\text { Descritivo e } \\
\text { analítico/ IV }\end{array}$ & $\begin{array}{l}\text { Idade: } 20-47 \text { anos } \\
(31,0 \text { anos) } \\
\% \text { mulheres* }\end{array}$ & $\begin{array}{l}\text { El Salvador, Honduras, } \\
\text { Nicarágua, México }\end{array}$ \\
\hline 36 & Salas, Reyes \& Reyes (2010) & Chile & $\begin{array}{l}\text { Identificar fatores de risco e comportamentos de prevenção do HIV/ } \\
\text { AIDS entre a população latino-americana residente no Chile }\end{array}$ & Etnografia/ IV & $\begin{array}{l}\text { Idade: } 17 \text { - } 44 \text { anos } \\
58,3 \% \text { mulheres }\end{array}$ & $\begin{array}{l}\text { Peru, Equador, Colômbia, } \\
\text { Argentina e Bolívia }\end{array}$ \\
\hline 37 & Taylor et al. (2014) & EUA & $\begin{array}{l}\text { Usar as comunidades vinculadas à migração de Santo Domingo, } \\
\text { República Dominicana, e Nova York, para determinar o impacto da } \\
\text { mobilidade geográfica no engajamento na assistência ao HIV e adesão } \\
\text { ao tratamento }\end{array}$ & Etnografia/ IV & $\begin{array}{l}\text { Idade }: \geq 21 \text { anos } \\
54 \% \text { mulheres }\end{array}$ & República Dominicana \\
\hline 38 & Valdez (2001) & EUA & $\begin{array}{l}\text { Explorar como a cultura guiou as práticas de saúde e para identificar } \\
\text { comportamentos de busca de saúde e atividades populares de cuidado de } \\
\text { mulheres hispânicas HIV positivo }\end{array}$ & Teoria fundamentada/I & $\begin{array}{l}\text { Idade: } 19-40 \text { anos } \\
\text { (31,0 anos) } \\
\% \text { mulheres* }\end{array}$ & México, Porto Rico \\
\hline 39 & Wilson \& McQuiston (2006) & EUA & $\begin{array}{l}\text { Compreender atitudes das mulheres imigrantes mexicanas em relação ao } \\
\text { planejamento de sua gravidez e os fatores que influenciam suas } \\
\text { preferências de fertilidade }\end{array}$ & $\begin{array}{l}\text { Descritivo e } \\
\text { analítico/ IV }\end{array}$ & $\begin{array}{l}\text { Idade: } 18-45 \text { anos } \\
\% \text { mulheres* }\end{array}$ & México \\
\hline \multicolumn{7}{|c|}{ Estudos de métodos mistos } \\
\hline 40 & $\begin{array}{l}\text { Coleman-Minahan \& Samari } \\
\text { (2018) }\end{array}$ & EUA & $\begin{array}{l}\text { Examinar as relações pai-filha e a saúde sexual dos adolescentes em } \\
\text { famílias de imigrantes de origem mexicana }\end{array}$ & $\begin{array}{l}\text { História de vida, } \\
\text { Longitudinal/ } \\
\text { IV }\end{array}$ & $\begin{array}{l}\text { Idade: } 27-41 \text { anos } \\
\% \text { mulheres* }\end{array}$ & México \\
\hline
\end{tabular}

Notas: $*_{n}=100 \%$ mulheres; $* * n=100 \%$ mulheres transexuais; NI = Não informado; NE= Nível de Evidência. Média da idade entre parênteses. Fonte: Autores. 
Dentre os temas relacionados à SSR, a violência se mostrou de forma transversal. A situação de SSR das migrantes latino-americanas está apresentada de acordo com as categorias formadas por similaridade e frequência temática (Quadro 3).

Quadro 3. Síntese da análise de conteúdo.

\begin{tabular}{ll}
\hline Categorias & Situação de saúde sexual e reprodutiva
\end{tabular}

$>$ Estresse, medo, preocupações com a deportação (Rocha-Jiménez, Brouwer, Silverman, Morales-Miranda \& Goldenberg, 2017) e incerteza associados a repercussões físicas e psicológicas (Salas, G. C. Reyes \& M. S. Reyes, 2010; Ceballos \& Palloni, 2010; Moreno, 2007; McDonald, Suellentrop, Paulozzi \& Morrow, 2008; Arcos, Vollrath, Sánchez \& Bailey, 2018; Grace et al., 2020); o estresse por ser mulher (Valdez, 2001);

Processo migratório e estabelecimento no país de acolhimento: implicações sobre a saúde e bem-estar

$>$ A imigração favoreceu riscos, e reduziu a possibilidade de autocuidado em saúde sexual e reprodutiva (Salas et al., 2010); Maior exposição e vulnerabilidade a traumas, violência sexual e comércio sexual (Fortuna et al., 2019; Santos, Drezett \& Alves, 2015; Salas et al., 2010); vitimização por violência no processo migratório (FernándezNiño et al., 2019); propensão a sofrer de violência por parceiro íntimo no país de acolhimento (Moreno, 2007; Salas et al., 2010; Grace et al., 2020) e coerção reprodutiva (Grace et al., 2020);

$>\quad$ Falta da rede de apoio no país de acolhimento (Salas et al., 2010; Grace et al., 2020; Ceballos \& Palloni, 2010) ou rede de apoio caracterizada por pessoas nascidas no mesmo país que a imigrante (Althoff et al., 2017; Waldman, 2011);

$>\quad$ As questões de saúde reprodutiva como secundárias na imigração em detrimento das condições socioeconômicas no país desconhecido (Matsuda et al.,2014); e precariedade socioeconômica (Moreno, 2007; Arcos et al., 2018; Wilson \& McQuiston, 2006; Santos et al., 2015; Coleman-Minahan \& Samari, 2018; Wilson, 2008).

$>$ Foram apontados como limitações do acesso aos serviços de saúde: falta de domínio do idioma (Waldman, 2011; Valdez, 2001), discriminação, preconceito, exclusão social e xenofobia por parte dos profissionais dos serviços de saúde (Salas et al., 2010; Waldmann, 2011; Martes \& Faleiros, 2013; Valdez, 2001), o status migratório associado à precariedade do atendimento à saúde sexual e reprodutiva (Salas et al., 2010; Fortuna et. al., 2019; White, Blackburn, Manzella, Welty \& Menachemi, 2014; Madi, Cassanti \& Silveira, 2009; Leal-Mateos, 2008; Joyce Bauer, Minkoff \& Kaestner, 2001); desconhecimento e falta de informação sobre o sistema de saúde do país de acolhimento (Arcos et al., 2018; Loue, Cooper \& Lloyd, 2005; Bahamondes et al., 2020), a necessidade de ausentar-se do trabalho para consultas (Waldman, 2011); barreiras institucionais e estruturais como falta de testes e medicamentos (Arcos et al., 2018; Rocha-Jiménez et al., 2017); baixa vinculação ao sistema de saúde (FernándezNiño et al., 2019);

Acesso aos serviços de saúde: barreiras e facilidades

$>\quad$ Menor acesso aos serviços de saúde sexual e reprodutiva de mulheres indígenas, pelas que não estavam gestantes (Martes \& Faleiros, 2013) ou que não pariram após a migração (Bahamondes et al., 2020); Falta de comunicação com os serviços de atendimento em situações de violência sexual (Santos et al. 2015); e a exigência de documentos para o atendimento nos serviços de saúde (Rocha-Jiménez et al., 2017; Waldman, 2011); Políticas anti-imigratórias (Spetz, Baker, Phibbs, Pedersen \& Tafoya, 2000; Joyce et al., 2001; Fuentes-Afflick et al., 2006; Fullerton, Nelson, Shannon \& Bader, 2004; Loue et al., 2005);

$>\quad$ Algumas facilidades no acesso à saúde foram observadas como: transporte para o local de atendimento (Fullerton et al., 2004); gratuidade de serviços de saúde pública, e material de educação em saúde sexual e reprodutiva no idioma da imigrante (Martes \& Faleiros, 2013); obtenção de atendimento sem a verificação do status legal (Martes \& Faleiros, 2013; Fernández-Niño et al., 2019); oportunidades de atendimento nos países vizinhos em regiões de fronteira (Fullerton et al., 2004); cartão de saúde como vantagem para garantir serviços de saúde sexual e reprodutiva (Rocha-Jiménez et al., 2017; Martes \& Faleiros, 2013); E o acesso à saúde no país de acolhimento diminuiu a necessidade de cruzar a fronteira para obtenção de serviços de saúde sexual e reprodutiva (Potter, White, Hopkins, Amastae \& Grossman, 2010; Fullerton et al., 2004).

> Motivos para evitar a gravidez das mulheres influenciados pela imigração, falta de apoio social ou questões socioeconômicas (Wilson \& McQuiston, 2006); adiar a gravidez por experiência de violência física, sexual e emocional (Grace et al., 2020; Wilson \& McQuiston, 2006);

$>\quad$ Diferenças de gênero sobre a responsabilidade pela contracepção (Salas et al., 2010; Matsuda et al., 2014); falta de autonomia sobre a decisão reprodutiva, sabotagem do parceiro como forma de interferir na contracepção levaram as mulheres a esconder ou mentir o sobre método contraceptivo (Grace et al., 2020), desincentivo do parceiro para uso do método contraceptivo (Biggs, Combellick, Arons \& Brindis, 2013);
Condicionantes ao atendimento das necessidades de planejamento reprodutivo e contracepção

$>\quad$ Baixo uso de serviços de planejamento reprodutivo no país de acolhimento (Martes \& Faleiros, 2013; Bahamontes et al., 2020) e possíveis desconhecimentos relacionados à falta de informação sobre saúde sexual e reprodutiva entre adolescentes e adultas, e sobre os recursos do país de acolhimento (Jones, Bond, Gardner \& Hernandez, 2002; Madi et al., 2009; Biggs et al., 2013; Wilson, 2009);

$>\quad$ Maior propensão de adolescentes e adultas migrantes a retornar às consultas de planejamento reprodutivo após o parto (Jones, Kubelka \& Bond, 2001; Jones et al., 2002; Wilson, 2009);

$>\quad$ As migrantes não buscavam serviços de saúde para obtenção de método contraceptivo, comprando-os em farmácias (Salas et al., 2010; Potter et al., 2010), ou obtiveram por envio do país de origem ou cruzando a fronteira (Grace et al., 2020; Potter et al., 2010);

> Relatos do não uso de contraceptivos por dificuldades de custeio (McDonald et al., 2008; Wilson, 2009); as mulheres não conseguiram obter anticoncepcionais nos serviços de saúde ou não conseguiram qualquer outra forma de contracepção (Bahamondes et al., 2020);

$>\quad$ Imigrantes relataram não ter controle sobre o ciclo menstrual (Madi et al., 2009).

$>$ As imigrantes que tinham menor renda eram mais propensas a engravidar e a casar (Wilson, 2008); Barreiras socioeconômicas, culturais e falta de oportunidades levaram à gravidez na adolescência (Minnis et al., 2013; Biggs et al., 2013; McDonald et al., 2008);

Antecedentes e implicações na gravidez
$>$ Instabilidade dentro da família, desorganização doméstica devido à separação dos pais ou divórcio influenciaram na saúde reprodutiva no país de acolhimento (Minnis et al., 2013);

$>$ Barreiras legais e linguísticas inibiram aspirações e expectativas educacionais de adolescentes resultaram em 


\begin{tabular}{|c|c|c|}
\hline & $>$ & $\begin{array}{l}\text { gravidez precoce (Minnis et al., 2013); } \\
\text { Mulheres adultas durante a gravidez apresentaram risco de aborto clandestino, relação conjugal instável devido a } \\
\text { sua condição de imigrante (Grace et al., 2020; Arcos et al., 2018); } \\
\text { As mulheres relataram violência na gestação por parte do companheiro ou outros membros do grupo social de } \\
\text { apoio (Fernández-Niño et al., 2019). }\end{array}$ \\
\hline $\begin{array}{l}\text { Sexualidade, } \\
\text { prevenção e } \\
\text { tratamento de HIV e } \\
\text { IST }\end{array}$ & $>$ & $\begin{array}{l}\text { Mulheres imigrantes apresentaram baixa prevalência de vacinação e rastreio de hepatite B (Kilmer, Barker, Ly \& } \\
\text { Jiles, 2019); menor frequência de testagem para HIV e sífilis (Fernández-Niño et al., 2019); Chances aumentadas } \\
\text { de comportamento sexual de risco, como parceiros casuais e relação sexual desprotegida (Salas et al., 2010; } \\
\text { Nuttbrock \& Hwahng, 2017; Loue, Cooper \& Fiedler, 2003; Althoff et al., 2017) } \\
\text { Adolescentes em famílias de imigrantes que foram separadas de seus pais tiveram maior risco de início precoce da } \\
\text { vida sexual (Coleman-Minahan \& Samari, 2018); } \\
\text { Mulheres adultas apresentaram vulnerabilidade à violência por parceiro íntimo relacionada à infecção pelo HIV } \\
\text { (Moreno, 2007; Fortuna et. al., 2019); alto risco de transmissão do HIV (Loue et al., 2003); maior propensão a } \\
\text { adquirir HIV/IST (Nuttbrock \& Hwahng, 2017); } \\
\text { Mulheres transexuais imigrantes estiveram mais envolvidas com o comércio sexual (Nuttbrock \& Hwahng, 2017); } \\
\text { As imigrantes revelaram desconhecimentos quanto à transmissão e prevenção de HIV/IST (Salas et al., 2010); } \\
\text { Falta de acesso à moradia digna e dificuldades de experiência sexual protegida (Salas et al., 2010); } \\
\text { Limitações no fornecimento de medicamentos para imigrantes com HIV que viajaram para fora do país de } \\
\text { acolhimento (Taylor et al., 2014); alta prevalência em queixas e sintomas de infecções sexualmente transmissíveis e } \\
\text { consequente consulta para atendimento de saúde sexual (Bahamondes et al., 2020). }\end{array}$ \\
\hline $\begin{array}{l}\text { Cuidados no pré- } \\
\text { natal, parto, } \\
\text { puerpério e } \\
\text { cuidados } \\
\text { obstétricos de } \\
\text { emergência }\end{array}$ & $\begin{array}{l}> \\
D \\
> \\
> \\
D\end{array}$ & $\begin{array}{l}\text { As mulheres procuraram e receberam cuidados pré-natais no país de acolhimento (Waldman, 2011; Ceballos \& } \\
\text { Palloni, 2010); disparidades das imigrantes no atendimento quanto ao número de consultas e início do pré-natal } \\
\text { (Fuentes-Afflick et al., 2006; Fernández-Niño et al., 2019; Leal-Mateos, 2008; Batista, Gugelmin \& Muraro, 2018; } \\
\text { McDonald et al., 2008; Spetz et al., 2000); } \\
\text { Adolescentes apresentaram risco de cuidados pré-natais inadequados (Fuentes-Afflick et al., 2006); } \\
\text { Influências étnicas nos padrões do cuidado pré-natal; as mulheres relataram cruzar a fronteira para receber cuidados } \\
\text { de saúde de curandeiros comunitários tradicionais como parteiras (Fullerton et al., 2004); } \\
\text { Fatores socioeconômicos como barreiras associadas ao acesso aos cuidados pré-natais (Fuentes-Afflick et. al, 2006); } \\
\text { Percepções das mulheres sobre os fatores que afetaram positivamente no atendimento do pré-natal: profissionais } \\
\text { amigáveis, que falavam o idioma; e acesso a exames (Fullerton et al., 2004); } \\
\text { As mulheres cruzaram a fronteira para receber alguns cuidados pré-natais e/ou para dar à luz a seus bebês no país de } \\
\text { acolhimento (Fullerton et al., 2004); a preferência pelo parto fora do país de origem indicada pela gratuidade, parto } \\
\text { sem dor, segurança e menor sofrimento foi relatado pelas imigrantes (Martes \& Faleiros, 2013); } \\
\text { As mulheres imigrantes tiveram mais complicações obstétricas como febre, sangramento e hipertensão (Forna, } \\
\text { Jamieson, Sanders \& Lindsay, 2003; Bahamondes et al., 2020); e as imigrantes estiveram menos propensas a } \\
\text { receber assistência pós-natal (Bahamondes et al., 2020). }\end{array}$ \\
\hline
\end{tabular}

Fonte: Autores.

\section{Discussão}

Por meio desta revisão, observou-se que a maior parte da produção referente ao processo migratório está voltada para a América do Norte (28), e que embora seja minoria, os artigos que tratam do fenômeno na América Central (2) e América do Sul (10) são mais recentes, revelando uma mudança geográfica no processo migratório, que pode estar associada as barreiras encontradas na América do Norte e a crise econômica por que passam os países da América Central e Sul.

Percebe-se ainda grande volume de artigos relacionados à migração decorrente de conflitos no Afeganistão, Iêmen, Síria, Sudão do Sul e Colômbia, que destacam vulnerabilidades de mulheres migrantes forçadas, e apontam que são particularmente expostas a riscos de exploração, violência sexual e comportamento sexual de risco por sobrevivência econômica, levando a um número crescente de gravidezes indesejadas, infecções sexualmente transmissíveis (ISTs), HIV, morte materna e precariedade em geral (Ivanova et al., 2018; Whelan \& Blogg, 2007; Ward \& Marsh, 2006; PAHO, 2019).

Revelou-se ainda que a migração e o processo de estabelecimento no país de acolhimento, por si só, já impactam diretamente na vida de mulheres que vivenciam essa experiência, estando relacionados a diversas questões que podem repercutir significativamente em seu estado físico e psicológico (Salas et al., 2010; Ceballos \& Palloni, 2010; Moreno, 2007; McDonald et al., 2008; Arcos et al., 2018; Grace et al., 2020; Fortuna et al., 2019). Além disso, a interrelação entre o gênero feminino, com a migração, representa maior causa de estresse, vulnerabilidade e insegurança nesse contexto (Valdez, 2001). As mulheres, durante o processo migratório, estiveram mais expostas, havendo relatos de violência, seja física e ou sexual, e comércio sexual (Salas et al., 2010; Fernández-Niño et al., 2019; Fortuna et al., 2019; Santos et al., 2015), além de vitimização por parceiro íntimo e coerção reprodutiva, no país de acolhimento (Moreno, 2007; Salas et al., 2010; Grace et al., 2020). 
Em relação aos corredores de migração latino-americanos, os artigos analisados nesta revisão, o faziam de forma superficial em relação à SSR, ou direcionados para recorte específico, com discussão restrita a este aspecto, sendo, portanto, pouco abrangente em relação à amplitude do assunto. Nesse sentido, não foram observados nos estudos grandes reflexões sobre os riscos de violação dos direitos de saúde sexual e reprodutiva para essa população, sendo necessária a soma de todos para realização dessa discussão mais direcionada ao tema.

Nos vários estudos sobre SSR de mulheres em idade reprodutiva analisados, não está claro como o processo de deslocamento é, em si mesmo, um determinante para estas questões. Por exemplo, sabe-se que o risco de gravidez cresce devido à falta de contraceptivos, à necessidade destes, à falta de acesso à proteção e ao maior risco de violência de gênero, incluindo estupro. Entretanto, nenhum estudo revisado examina em profundidade as experiências de mulheres que engravidam durante a viagem ou que migram ou viajam durante a gravidez, suas necessidades, cuidados e acesso.

Os achados da revisão são também apontados por Góes e Borges (2021), que destacam que nas condições de migração e refúgio, só pelo fato de serem mulheres, elas já ficam mais vulneráveis às violências baseada em gênero, sexual e psicológica, tráfico de pessoas, muitas vezes perpetradas pelos homens que as conduzem durante o trânsito, e outras formas de exploração. Além de desafios sociais, econômicos, culturais e políticos enfrentados nos países de acolhimento.

$\mathrm{Na}$ literatura, observam-se manuscritos que apontam a migração internacional e o refúgio, como fatores que impactam diretamente nas necessidades relativas à saúde e bem-estar de mulheres e adolescentes. Referem inclusive à migração como um determinante para problemas de saúde desta população devido aos riscos enfrentados nesse processo. Apontam ainda as consequências imediatas para a saúde pública, efeitos a longo prazo e intergeracionais para o desenvolvimento social e bem-estar de migrantes, em particular mulheres (Starrs et al 2018; Wickramage \& Annunziata, 2018).

No que se refere ao acesso aos serviços de saúde, os artigos revisados apontaram um histórico de condições dificultadoras e facilitadoras. Um exemplo é que diversos estudos publicados no início dos anos 2000, nos Estados Unidos da América (EUA), reportavam leis de restrição do acesso de imigrantes aos serviços de saúde, incluindo SSR, em geral, relacionados ao atendimento pré-natal (Spetz et al., 2000; Joyce et al., 2001; Fuentes-Afflick et al., 2006; Fullerton et al., 2004; Loue et al., 2005), e contraceptivos (White et al., 2014), consequentemente diminuindo o acesso e reduzindo o financiamento da assistência. Além disso, nas políticas de imigração nesse país, alguns autores (Coleman-Minahan \& Samari, 2018), citaram casos de deportação de pais das imigrantes adolescentes, com reflexos na perda de suporte e proteção familiar, as expondo a maiores riscos, como iniciação sexual precoce. Vale destacar que a discussão de políticas anti-imigração foi alvo de publicações ao longo das duas décadas seguintes, como revelado em outros estudos realizados (White et al., 2014; Fortuna et al., 2019). White et al. (2014) acrescentaram a esse histórico que serviços anticoncepcionais, exames ginecológicos, imunizações, testes ou tratamentos para doenças transmissíveis foram serviços menos utilizados pelas migrantes após a Lei Alabama Law, aumentando os riscos de resultados desfavoráveis para SSR.

Tendo em vista que políticas anti-imigração nos países de acolhimento geram impactos significativos em comunidades de imigrantes, para ampliar a discussão desta revisão, encontrou-se manuscrito (Menjívar \& Abrego, 2012) que relata que a implementação dessas políticas constitui uma forma de violência, denominada mais precisamente de violência legal. Na contramão de medidas favoráveis à saúde e ao bem-estar de mulheres e adolescentes migrantes, essas medidas mostram-se como excludentes, mas também geram efeitos violentos para os imigrantes como um todo, afetando a vida cotidiana e a curto e longo prazo.

Ainda discutindo o acesso à saúde das mulheres migrantes, os estudos analisados na revisão revelaram restrições que advém de políticas públicas, e de processos de trabalho. Em países onde a assistência é mediada por elegibilidade de seguros 
de saúde, filantropia ou pagamento privado (Fortuna et al., 2019), predomina a barreira de acesso, já nos de sistemas de saúde universais, observa-se a garantia de acesso, por vezes até desconsiderando o status migratório da mulher. No entanto, a maneira como a assistência é prestada às migrantes, pode constituir outra barreira, contribuindo para uma vinculação frágil com os serviços de saúde, ou até mesmo para seu afastamento (Salas et al., 2010; Fortuna et. al., 2019; White et al., 2014; Madi et al., 2009; Leal-Mateos, 2008; Joyce et al., 2001; Fernández-Niño et al., 2019), visto que em alguns serviços, dentre outros problemas, foi apontada uma assistência permeada por postura discriminatória e xenofóbica nas práticas da equipe de saúde (Waldmann, 2011; Martes \& Faleiros, 2013; Salas et al., 2010). Estes achados vão ao encontro de outros manuscritos da literatura, que apontam que tais condições repercutem em maior precarização da situação de saúde dessa população (O’neil et al.,; Rocha-Jiménez et al., 2017; Filler et al., 2020).

Segundo O’neil et al. (2016) existem evidências consideráveis e persistentes relacionadas às iniquidades de gênero, na qualidade da atenção à saúde em países com recursos menores e maiores. Para Filler et al. (2020), o atendimento a essa população apresenta questões relacionadas a cultura, raça, etnia, religião, gênero e orientação sexual e refere-se a uma assistência que necessita ser adequada às crenças de saúde das migrantes sobre sua doença e suas causas. Todavia, a interpretação das questões de saúde muitas vezes se resume ao contexto biomédico, em vez de biopsicossocial.

Embora em poucos estudos desta revisão, destaca-se que foram apontadas algumas estratégias para facilitar o acesso da mulher migrante à assistência em saúde, dentre estas, a garantia de transporte para o local de atendimento e da obtenção do cartão nacional de saúde (Fullerton et al., 2004; Martes \& Faleiros, 2013; Rocha-Jiménez et al., 2017).

Além disso, nos muitos estudos analisados para escolha dos que comporiam essa revisão, chama-se a atenção que poucos discutem as necessidades, gestão de riscos e acesso, de forma específica por idade e identidade de gênero dos entrevistados, apesar de tais variáveis serem de fundamental relevância para determinar os desfechos desse fenômeno para os sujeitos em migração. Este fato implicou na redução do número de artigos selecionados uma vez que o recorte do estudo é para mulheres, mas os resultados destes seguiram não dissociados por idade. Portanto, poucos dos estudos analisados investigaram as condições das migrantes e refugiadas adolescentes em separado das mulheres adultas, restringindo-se a discussão a gravidez na adolescência e uso de contraceptivos.

No geral, os artigos descrevem seus achados sobre SSR, para população migrante adolescente e adulta de forma unificada. Assim, chama-se atenção para o fato de que um número bastante elevado de adolescentes em deslocamento enfrenta riscos específicos, moldados por características intersetoriais de gênero e idade (acrescidos de outros como raça, etnia, sexualidade e classe). Essas características podem desempenhar um forte papel na influência do comportamento para lidar ou evitar riscos em deslocamento, e dos mecanismos de enfrentamento. Ampliando essa discussão com outro achado da literatura, encontrou-se que a população adolescente a nível global enfrenta uma tripla carga de doenças, sendo uma delas as doenças transmissíveis relacionadas à saúde reprodutiva (Liang et al. 2019). Dessa forma, percebe-se ainda mais a necessidade de investigações sobre SSR específica dessa população.

Outro achado relevante, foi que vários estudos sobre SSR de mulheres em idade reprodutiva não deixam claro como o processo de deslocamento é, em si mesmo, um determinante para esta questão. Por exemplo, sabe-se que o risco de gravidez cresce devido à falta de contraceptivos, à necessidade destes, à falta de acesso à proteção e ao maior risco de violência de gênero, incluindo estupro. Entretanto, nenhum estudo revisado examina em profundidade as experiências de mulheres que engravidam durante a viagem ou que migram ou viajam durante a gravidez em relação às suas necessidades, cuidados e acesso.

Além disso, nos estudos encontrados, poucos dão voz às mulheres nessa condição, para que identifiquem suas necessidades de SSR por conta própria, sendo que os que o fizeram, viram a contracepção assumir maior importância decorrente de um desejo compreensível de evitar a gravidez. A violência, em todas as suas formas, também esteve presente de forma transversal, mas não foi foco de nenhum estudo, não havendo aprofundamentos sobre o tema. 
Assim, analisando os artigos que compõem esta revisão, embora apontem diversas dificuldades enfrentadas pelas mulheres no processo de migração, em relação à SSR, percebe-se uma fragilidade no aprofundamento das discussões no que diz respeito às estratégias de enfrentamento que utilizam quando expostas a estas situações. Assim, persiste essa lacuna em relação às informações disponíveis sobre como se organizam para minimizar estes danos, as causas, o quanto de fato são impactadas por essas condições, e consequentemente não apontam possíveis soluções.

A educação em saúde sexual também foi um tema abordado nos estudos incluídos na revisão, a maioria dos artigos apontou falta de informação ou desconhecimento das migrantes em relação à SSR (Biggs et al., 2013; Bahamondes et al., 2020; Minnis et al., 2013), assim como, desconhecimentos sobre o sistema de saúde do país de acolhimento (Arcos et al., 2018; Loue et al., 2005; Bahamondes et al., 2020). Para Hegde et al., (2012), esta falta de informação sobre o direito de acesso a cuidados de saúde e medicamentos, bem como o custo de serviços privados, independentemente do status de migração, podem levar algumas mulheres migrantes a renunciar os cuidados de SSR, especialmente cuidados preventivos, mesmo em situações de risco extremo, como complicações por indução ou abortos espontâneos.

Cumpre destacar que, quando analisados os riscos relacionados à sexualidade, prevenção e tratamento de HIV e ISTs de mulheres migrantes, a população cisgênero e de transexuais foi apontada em diversos estudos como ainda mais impactadas nesse processo. Para além das barreiras estruturais, esta população enfrenta o estigma social (prevalente nas comunidades latino-americanas), tanto no país de origem como no país de destino das migrantes, refletindo no adiamento da testagem para HIV e postergando a busca de cuidados de saúde. Tal fato, gera prejuízos imensuráveis para essas mulheres, pois a descoberta da doença acaba ocorrendo em estágios avançados (Taylor et al., 2014; Nuttbrock \& Hwahng, 2017; Moreno, 2007).

Além disso, observou-se nos estudos da revisão, que a orientação sexual esteve fortemente relacionada ao trabalho sexual e à HIV/IST (Nuttbrock \& Hwahng, 2017), a menor frequência na testagem para HIV e sífilis (Fernandez-Niño et al., 2019), e a alta prevalência em queixas e sintomas de IST, consequentemente, levando a consultas para atendimento de saúde sexual (Bahamondes et al., 2020). No caso de mulheres transexuais, foi observado que tais fatores culminaram num elevado risco para o estilo de vida, associado ao trabalho sexual, e comportamentos sexuais de alto risco que levam a HIV/IST (Nuttbrock \& Hwahng, 2017).

\section{Considerações Finais}

Com esta revisão observou-se que a migração de mulheres nos corredores da América Central e do Sul tem sido marcada por considerável crescimento, todavia, ainda se encontra pequeno o volume de produção para explicar o fenômeno nesses territórios. Revelou-se ainda que a migração e o processo de estabelecimento no país de acolhimento, por si só, já impactam diretamente na vida de mulheres que vivenciam essa experiência, estando relacionados a diversas questões que podem repercutir significativamente em seu estado físico e psicológico.

Destaca-se que nos muitos estudos analisados para escolha de artigos para compor essa revisão, poucos discutiam as necessidades, gestão de riscos e acesso da população migrante com distinção por idade e identidade de gênero, apesar de tais variáveis serem de fundamental relevância para determinar os desfechos desse fenômeno para os sujeitos migrantes. Considerando ainda os artigos escolhidos para revisão, no geral descrevem seus achados sobre SSR, para população migrante adolescente e adulta de forma unificada, sendo que as adolescentes em deslocamento enfrentam riscos específicos, moldados por características intersetoriais de gênero e idade.

Os artigos analisados em relação à SSR, não apresentaram grandes reflexões sobre os riscos para violação dos direitos de saúde sexual e reprodutiva de mulheres, não deixaram claro como o processo de deslocamento é, em si mesmo, um determinante para estas questões. Além disso, nos estudos encontrados, poucos dão voz às mulheres nessa condição, para que 
identifiquem suas necessidades de SSR por conta própria, sendo que os que o fizeram, viram a contracepção assumir maior importância decorrente de um desejo compreensível de evitar a gravidez.

No que se refere ao acesso aos serviços de saúde, os artigos revisados apontaram um histórico de condições dificultadoras que incluem desde políticas restritivas, redução no financiamento da assistência, postura discriminatória e xenofóbica nas práticas da equipe de saúde, e facilitadoras como a garantia de transporte para o local de atendimento e facilitação para obtenção de documentação para acesso.

Embora apontem diversas dificuldades enfrentadas pelas mulheres no processo de migração, em relação a SSR, percebe-se uma fragilidade no aprofundamento das discussões no que diz respeito as estratégias de enfrentamento que utilizam quando expostas a estas situações. Assim, persiste essa lacuna em relação as informações disponíveis sobre como se organizam para minimizar estes danos, as causas, o quanto de fato são impactadas por essas condições, e consequentemente quais seriam as possíveis soluções.

Diante disso, é fundamental que trabalhos futuros abordem de forma mais individualizada as origens, as vulnerabilidades e as necessidades das populações femininas migrantes, para que a compreensão dessas trajetórias e a implementação de medidas, governamentais ou não, que facilitem esses deslocamentos, seja potencializada, preservando o bem-estar dessas mulheres e de suas famílias.

\section{Referências}

Alarcão, V., Stefanovska-Petkovska, M., Virgolino, A., Santos, O., \& Costa, A. (2021). Intersections of Immigration and Sexual/Reproductive Health: An Umbrella Literature Review with a Focus on Health Equity. Social Sciences, 10(2), 63. https://doi.org/10.3390/socsci10020063

Althoff, M. D., Theall, K., Schmidt, N., Hembling, J., Gebrekristos, H. T., Thompson, M. M., Muth, S. Q., Friedman, S. R., \& Kissinger, P. (2017). Social Support Networks and HIV/STI Risk Behaviors Among Latino Immigrants in a New Receiving Environment. AIDS and Behavior, 21 (12), 3607-3617. https://doi.org/10.1007/s10461-017-1849-8

Arcos, E., Vollrath, A., Sánchez, X., \& Bailey, C. (2018). Motherhood inmigrant women in Chile: A qualitative study. Midwifery, 66, 182-186. https://doi.org/10.1016/j.midw.2018.08.008

Bahamondes, L., Laporte, M., Margatho, D., de Amorim, H. S. F., Brasil, C., Charles, C. M., Becerra, A., \& Hidalgo, M. M. (2020). Maternal health among Venezuelan women migrants at the border of Brazil. BMC Public Health, 20(1), 1-8. https://doi.org/10.1186/s12889-020-09912-x

Bardin, L. (2011). Análise de conteúdo. São Paulo: Edições 70. Brasil.(2014a). Manual de enfrentamento à violência contra a pessoa idosa. Brasília: Secretaria de Direitos Humanos da Presidência da República.

Batista, D. R. R., Gugelmin, S. A., \& Muraro, A. P. (2018). Prenatal follow-up of Haitian and Brazilian women in Mato Grosso. Revista Brasileira de Saúde Materno Infantil, 18, 317-326. https://doi.org/10.1590/1806-93042018000200005

Bautista, C. T., Mosquera, C., Serra, M., Gianella, A., Avila, M. M., Laguna-Torres, V., Carr, J. K., Montano, S. M., \& Sanchez, J. L. (2007). Immigration Status and HIV-risk Related Behaviors among Female Sex Workers in South America. AIDS and Behavior, 12(2), 195-201. https://doi.org/10.1007/s10461007-9270-3

Biggs, M. A., Combellick, S., Arons, A., \& Brindis, C. D. (2013). Educational Barriers, Social Isolation, and Stable Romantic Relationships Among Pregnant Immigrant Latina Teens. Hispanic Health Care International, 11(1), 38-46. https://doi.org/10.1891/1540-4153.11.1.38

Ceballos, M., \& Palloni, A. (2010). Maternal and infant health of Mexican immigrants in the USA: the effects of acculturation, duration, and selective return migration. Ethnicity\& Health, 15(4), 377-396. https://doi.org/10.1080/13557858.2010.481329

Coleman-Minahan, K., \&Samari, G. (2018). "He supported me 100\%": Mexican-immigrant fathers, daughters, and adolescent sexual health. Ethnicity \& Health, 25(4), 560-579. https://doi.org/10.1080/13557858.2018.1439897

Dutra, D. (2013). Mulheres, migrantes, trabalhadoras: a segregação no mercado de trabalho. REMHU: Revista Interdisciplinar Da Mobilidade Humana, 21, 177-193. https://www.scielo.br/j/remhu/a/SHpdGQt7Mdq3rw4KWvZkyzn/?format=html\&lang=pt

Fernández-Niño, J. A., Rojas-Botero, M. L., Bojorquez-Chapela, I., Giraldo-Gartner, V., Sobczyk, R. A., Acosta-Reyes, J., Flórez-García, V., Rodríguez, D. A., Fernández-Niño, J. A., Rojas-Botero, M. L., Bojorquez-Chapela, I., Giraldo-Gartner, V., Sobczyk, R. A., Acosta-Reyes, J., Flórez-García, V., \& Rodríguez, D. A. (2019). Situación de salud de gestantes migrantes venezolanas en el Caribe colombiano: primer reporte para una respuesta rápida em Salud Pública. Revista de La Universidad Industrial de Santander. Salud, 51(3), 208-219. https://doi.org/10.18273/revsal.v51n3-2019004

Filler, T., Jameel, B., \& Gagliardi, A. R. (2020). Barriers and facilitators of patient Centered care for immigrant and refugee women: a scoping review. BMC Public Health, 20(1), 1-12. https://doi.org/10.1186/s12889-020-09159-6 
Forna, F., Jamieson, D. J., Sanders, D., \& Lindsay, M. K. (2003). Pregnancy outcomes in foreign-born and US-born women. International Journal of Gynecology \& Obstetrics, 83(3), 257-265. https://doi.org/10.1016/s0020-7292(03)00307-2

Fortuna, L. R., Noroña, C. R., Porche, M. V., Tillman, C., Patil, P. A., Wang, Y., Markle, S. L., \& Alegría, M. (2019). Trauma, immigration, and sexual healthamong Latina women: Implications for maternal-child well-being and reproductive justice. Infant Mental Health Journal, 40(5), 640-658. https://doi.org/10.1002/imhj.21805

Fuentes-Afflick, E., Hessol, N. A., Bauer, T., O’Sullivan, M. J., Gomez-Lobo, V., Holman, S., Wilson, T. E., \&Minkoff, H. (2006). Use of Prenatal Care by Hispanic Women After Welfare Reform. Obstetrics \& Gynecology, 107(1), 151-160. https://doi.org/10.1097/01.AOG.0000191299.24469.1b

Fullerton, J. T., Nelson, C., Shannon, R., \&Bader, J. (2004). Prenatalv Care in the Paso del Norte Border Region. Journal of Perinatology, 24(2), 62-71. https://doi.org/10.1038/sj.jp.7211028

Gabaccia, D. R. (2016). Feminization of Migration. The Wiley Blackwell Encyclopedia of Gender and Sexuality Studies, 1-3. https://doi.org/10.1002/9781118663219.wbegss732

Góes, E. D. A. de, \& Borges, A. V. B. (2021). Entre a cruz e a espada: múltiplas violências contra mulheres refugiadas. SER Social, 23 (49), 318-337. https://doi.org/10.26512/sersocial.v23i49.35871

Grace, K. T., Alexander, K. A., Jeffers, N. K., Miller, E., Decker, M. R., Campbell, J., \& Glass, N. (2020). Experiences of Reproductive Coercion Among Latina Women and Strategies for Minimizing Harm: "The Path Makes Us Strong." Journal of Midwifery \& Women's Health, 65(2), 248-256. https://doi.org/10.1111/jmwh.13061

Gunn, W. (2014). Mendeley: Enabling and understanding scientific collaboration. Information. Services \& Use, 34(1-2), 99-102. https://doi.org/10.3233/isu140738

Hegde, S., Hoban, E., \& Nevill, A. (2011). Unsafe Abortion as a Birth Control Method. Asia Pacific Journal of Public Health, 24(6), 989-1001. https://doi.org/10.1177/1010539511409024

International Organization for Migration (2021). Key MigrationTerms..https://www.iom.int/key-migration-terms\#Refugee-mandate

Ivanova, O., Rai, M., \&Kemigisha, E. (2018). A Systematic Review of Sexual and Reproductive Health Knowledge, Experiences and Access to Services among Refugee, Migrant and Displaced Girls and Young Women in Africa. International Journal of Environmental Research and Public Health, 15(8), 1583. https://doi.org/10.3390/ijerph15081583

Jones, M. E., Bond, M. L., Gardner, S. H., \& Hernandez, M. C. (2002). Acculturation level and family-planning patterns of Hispanic immigrant women. MCN: The American Journal of Maternal/Child Nursing, 27(1), 26-32. https://doi.org/10.1097/00005721-200201000-00009

Jones, M. E., Kubelka, S., \& Bond, M. L. (2001). Acculturation Status, Birth Outcomes, and Family Planning Compliance Among HispanicTeens. The Journal of School Nursing, 17(2), 83-89. https://doi.org/10.1177/105984050101700205

Joyce, T., Bauer, T., Minkoff, H., \& Kaestner, R. (2001). Welfare Reform and the Perinatal Health and Health Care Use of Latino Women in California, New York City, and Texas. American Journal of Public Health, 91(11), 1857-1864. https://doi.org/10.2105/ajph.91.11.1857

Kilmer, G. A., Barker, L. K., Ly, K. N., \& Jiles, R. B. (2018). Hepatitis B Vaccination and Screening Among Foreign-born Women of Reproductive Age in the United States: 2013-2015. Clinica IInfectious Diseases, 68(2), 256-265. https://doi.org/10.1093/cid/ciy479

Leal-Mateos, M., \& Barboza-Retana, J. (2008). Características Del control prenatal y el parto de las mujeres inmigrantes atendidas en el Hospital "Dr. Rafael Ángel Calderón Guardia.” Acta Médica Costarricense, 50(2), 107-111. https://www.scielo.sa.cr/scielo.php?script=sci_arttext\&pid=S000160022008000200008

Liang, M., Simelane, S., Fortuny Fillo, G., Chalasani, S., Weny, K., Salazar Canelos, P., Jenkins, L., Moller, A.-B., Chandra-Mouli, V., Say, L., Michielsen, K., Engel, D. M. C., \&Snow, R. (2019). The State of Adolescent Sexual and Reproductive Health. Journal of Adolescent Health, 65(6, Supplement), S3-S15. https://doi.org/10.1016/j.jadohealth.2019.09.015

Liberati, A., Altman, D. G., Tetzlaff, J., Mulrow, C., Gøtzsche, P. C., Ioannidis, J. P. A., Clarke, M., Devereaux, P. J., Kleijnen, J., \&Moher, D. (2009). The PRISMA statement for reporting systematic reviews and meta-analyses of studies that evaluate healthcare interventions: explanation and elaboration. Journal of Clinical Epidemiology, 62(10), e1-e34. https://doi.org/10.1016/j.jclinepi.2009.06.006

Loue, S., Cooper, M., \& Fiedler, J. (2003). HIV Risk Among a Sample of Mexican and Puerto Rican Menand Women. Journal of Health Care for the Poor and Underserved, 14(4), 550-565. https://doi.org/10.1353/hpu.2010.0680

Loue, S., Cooper, M., \& Lloyd, L. S. (2005). Welfare and Immigration Reform and Use of Prenatal Care Among Women of Mexican Ethnicity in San Diego, California. Journal of Immigrant Health, 7(1), 37-44. https://doi.org/10.1007/s10903-005-1389-6

Madi, M. C. C., Cassanti, A. C., \& Silveira, C. (2009). Estudo das representações sociais sobre gestação em mulheres bolivianas no contexto da atenção básica em saúde na área central da cidade de São Paulo. Saúde e Sociedade, 18, 67-71. https://doi.org/10.1590/S0104-12902009000600011

Martes, A. C. B., \& Faleiros, S. M. (2013). Acesso dos imigrantes bolivianos aos serviços públicos de saúde na cidade de São Paulo. Saúde e Sociedade, 22, 351-364. https://doi.org/10.1590/S0104-12902013000200008

Matsuda, Y., McGrath, J. M., Knafl, G. J., Worthington, E. L., Jallo, N., \& Corona, R. (2014). Examining Relationship/Family Planning Factors and Sexual Relationship Power Among Immigrant Latino Couples in the United States. Hispanic Health Care International: The Official Journal of the National Association of Hispanic Nurses, 12(4), 161-173. https://doi.org/10.1891/1540-4153.12.4.161

McAuliffe, M., Khadria, B., \& Bauloz, C. (2019). World migration report 2020. Geneva: International Organization for Migration. 
McDonald, J. A., Suellentrop, K., Paulozzi, L. J., \& Morrow, B. (2007). Reproductive Health of the Rapidly Growing Hispanic Population: Data from the Pregnancy Risk Assessment Monitoring System, 2002. Maternal and Child Health Journal, 12(3), 342-356. https://doi.org/10.1007/s10995-007-0244-X

Mendoza, W., \& Miranda, J. J. (2019). La inmigración venezolana en el Perú: desafíos y oportunidades desde la perspectiva de la salud. Revista Peruana de Medicina Experimental Y Salud Pública, 36(3), 497-503. https://doi.org/10.17843/rpmesp.2019.363.4729

Menjívar, C., \& Abrego, L. J. (2012). Legal Violence: Immigration Law and the Lives of Central American Immigrants. American Journal of Sociology, 117(5), 1380-1421. https://doi.org/10.1086/663575

Metusela, C., Ussher, J., Perz, J., Hawkey, A., Morrow, M., Narchal, R., Estoesta, J., \& Monteiro, M. (2017). "In My Culture, We Don’t Know Anything About That": Sexual and Reproductive Health of Migrant and Refugee Women. International Journal of Behavioral Medicine, 24(6), 836-845. https://doi.org/10.1007/s12529-017-9662-3

Minnis, A. M., Marchi, K., Ralph, L., Biggs, M. A., Combellick, S., Arons, A., Brindis, C. D., \& Braveman, P. (2012). Limited Socioeconomic Opportunities and Latina Teen Childbearing: A Qualitative Study of Family and Structural Factors Affecting Future Expectations. Journal of Immigrant and Minority Health, 15(2), 334-340. https://doi.org/10.1007/s10903-012-9653-z

Minsart, A.-F., Englert, Y., \&Buekens, P. (2013). Naturalization of immigrants and perinatal mortality. European Journal of Public Health, 23(2), 269-274. https://doi.org/10.1093/eurpub/cks032

Miramontes, L., Pottie, K., Jandu, M. B., Welch, V., Miller, K., James, M., \& Roberts, J. H. (2015). Including migrant populations in health impact assessments. Bulletin of the World Health Organization, 93, 888-889. https://doi.org/10.2471/BLT.14.142315

Moreno, C. L. (2007). The Relationship Between Culture, Gender, Structural Factors, Abuse, Trauma, and HIV/AIDS for Latinas. Qualitative Health Research, 17(3), 340-352. https://doi.org/10.1177/1049732306297387

Nuttbrock, L. A., \& Hwahng, S. J. (2016). Ethnicity, Sex Work, and Incident HIV/STI Among Transgender Women in New York City: A Three Year Prospective Study. AIDS and Behavior, 21(12), 3328-3335. https://doi.org/10.1007/s10461-016-1509-4

O'Neil, T., Fleury, A., \& Foresti, M. (2016). Women on the move: Migration, gender equality and the 2030 Agenda for Sustainable Development. Federal Department of Foreign Affairs. Swiss Agency for Development and cooperation SDC.

Ouzzani, M., Hammady, H., Fedorowicz, Z., \& Elmagarmid, A. (2016). Rayyan - a web and mobile app for systematic reviews. Systematic Reviews, 5(1), 110. https://doi.org/10.1186/s13643-016-0384-4

Pan-American Health Organization (2019). Gender equality in addressing the causes and consequences of the health of migrant women, Techincal Report. https://www.paho.org/hq/index.php?option=com_docman\&view=download\&slug=international-womenday-2019-technical-note-eng\&Itemid=270\&lang=en

Potter, J. E., White, K., Hopkins, K., Amastae, J., \& Grossman, D. (2010). Clinic Versus Over-the-Counter Access to Oral Contraception: Choices Women Make Along the US-Mexico Border. American Journal of Public Health, 100(6), 1130-1136. https://doi.org/10.2105/ajph.2009.179887

Rivillas, J. C., Devia Rodriguez, R., Song, G., \& Martel, A. (2018). How do we reach the girls and women who are the hardest to reach? Inequitable opportunities in reproductive and maternal health care services in armed conflict and forced displacement settings in Colombia. PLOS ONE, 13(1), e0188654. https://doi.org/10.1371/journal.pone.0188654

Rocha-Jimenez, T., Brouwer, K. C., Salazar, M., Boyce, S. C., Servin, A. E., Goldenberg, S. M., Staines-Orozco, H., Vera-Monroy, R. B., \& Silverman, J. G. (2017). "He invited me and didn't ask anything in return" Migration and Mobility as Vulnerabilities for Sexual Exploitation among Female Adolescents in Mexico. International Migration, 56(2), 5-17. https://doi.org/10.1111/imig.12333

Salas, A. C., Reyes, C. G., \& Reyes, S. M. (2010). Salud sexual y reproductiva y migración latinoamericana en Chile: una aproximación cualitativa a las situaciones de riesgos y conductas de prevención del VIH SIDA. Cuad. méd.-soc. (Santiago de Chile), 322-339.

Santos, J., Drezett, J., \& Alves, A. de L. (2015). Características sociodemográficas de migrantes bolivianas com gestação decorrente de violência sexual atendidas em service público de referência para abortamento legal, São Paulo, Brasil, 2002-2014. Reprodução e Climatério, 30(1), 25-32. https://doi.org/10.1016/j.recli.2015.05.004

Spetz, J., Baker, L., Phibbs, C., Pedersen, R., \& Tafoya, S. (2000). The Effect of Passing an “Anti-immigrant” Ballot Proposition on the Use of Prenatal Care by Foreign-Born Mothers in California. Journal of Immigrant Health, 2(4), 203-212. https://doi.org/10.1023/a:1009540313141

Starrs, A. M., Ezeh, A. C., Barker, G., Basu, A., Bertrand, J. T., Blum, R., Coll-Seck, A. M., Grover, A., Laski, L., Roa, M., Sathar, Z. A., Say, L., Serour, G. I., Singh, S., Stenberg, K., Temmerman, M., Biddlecom, A., Popinchalk, A., Summers, C., \& Ashford, L. S. (2018). Accelerate progress-sexual and reproductive health and rights for all: report of the Guttmacher- Lancet Commission. The Lancet, 391(10140), 2642-2692. https://doi.org/10.1016/s01406736(18)30293-9

Stetler, C. B., Morsi, D., Rucki, S., Broughton, S., Corrigan, B., Fitzgerald, J., Giuliano, K., Havener, P., \& Sheridan, E. A. (1998). Utilization-focused integrative reviews in a nursing service. Applied Nursing Research, 11(4), 195-206. https://doi.org/10.1016/s0897-1897(98)80329-7

Sturrock, S., Williams, E., \& Greenough, A. (2020). Antenatal and perinatal outcomes of refugees in high income countries. Journal of Perinatal Medicine, 49(1), 80-93. https://doi.org/10.1515/jpm-2020-0389

Taylor, B. S., Reyes, E., Levine, E. A., Khan, S. Z., Garduño, L. S., Donastorg, Y., Hammer, S. M., Brudney, K., \& Hirsch, J. S. (2014). Patterns of Geographic Mobility Predict Barriers to Engagement in HIV Care and Antiretroviral Treatment Adherence. AIDS Patient Care and STDs, 28(6), 284-295. https://doi.org/10.1089/apc.2014.0028 
Research, Society and Development, v. 10, n. 16, e5101623698, 2021

(CC BY 4.0) | ISSN 2525-3409 | DOI: http://dx.doi.org/10.33448/rsd-v10i16.23698

United Nations Organization (2019). Department of Economic and Social Affairs, Population Division. International Migration 2019: Report. New York: United Nations.

United Nations Refugee Agency. (2015). Women on the Run: first hand accounts of refugees fleeing El Salvador, Guatemala, Honduras, and Mexico.UNHCR. https://www.unhcr.org/56fc31864.html

United Nations Refugee Agency. (2021). Venezuela situation. UNHCR. https://www.unhcr.org/venezuela-emergency.html

Valdez, M. del R. (2001). A Metaphor for HIV-Positive Mexican and Puerto Rican Women. Western Journal of Nursing Research, 23(5), 517-535. https://doi.org/10.1177/01939450122045357

Waldman, T. C. (2011). Movimentos migratórios sob a perspectiva do direito à saúde: imigrantes bolivianas em São Paulo. Revista de Direito Sanitário, 12(1), 90-114. https://doi.org/10.11606/issn.2316-9044.v12i1p90-114

Ward, J., \& Marsh, M. (2006). Sexual violence against women and girls in war and its aftermath: Realities, responses and required resources. In Symposium on Sexual Violence in Conflict and Beyond (Vol. 21, p. 23).

Whelan, A., \& Blogg, J. (2007). 'Halfway people': Refugee views of reproductive health services. Global public health, $2(4), 373-394$. https://doi.org/10.1080/17441690701267048

White, K., Blackburn, J., Manzella, B., Welty, E., \&Menachemi, N. (2014). Changes in Use of County Public Health Services Following Implementation of Alabama's Immigration Law. Journal of Health Care for the Poor and Underserved, 25(4), 1844-1852. https://doi.org/10.1353/hpu.2014.0194

Whittemore, R., \&Knafl, K. (2005). The integrative review: updated methodology. Journal of Advanced Nursing, 52(5), 546-553. https://doi.org/10.1111/j.1365-2648.2005.03621.x

Wickramage, K., \& Annunziata, G. (2018). Advancing health in migration governance, and migration in health governance. The Lancet, 392(10164), 25282530. https://doi.org/10.1016/S0140-6736(18)32855-1

Wilson, E. K. (2008). Acculturation and Changes in the Likelihood of Pregnancy and Feelings About Pregnancy Among Women of Mexican Origin. Women and Health, 47(1), 45-64. https://doi.org/10.1300/j013v47n01_03

Wilson, E. K. (2009). Differences in Contraceptive Use Across Generations of Migration Among Women of Mexican Origin. Maternal and Child Health Journal, 13(5), 641-651. https://doi.org/10.1007/s10995-008-0382-9

Wilson, E. K., \& McQuiston, C. (2006). Motivations for Pregnancy Planning Among Mexican Immigrant Women in North Carolina. Maternal and Child Health Journal, 10(3), 311-320. https://doi.org/10.1007/s10995-005-0055-x

World Health Organization. (2012). Health emergency and disaster risk management framework. http://www.who.int/hac/techguidance/preparedness/SRH_HERM_Policy_brief_A4.pdf?ua=1 